\title{
Understanding Allergic Fungal Rhino-Sinusitis
}

\author{
Sumit Sharma* \\ Department of E.N.T, Mayo Institute of Medical Sciences, India
}

Submission: February 01, 2018; Published: February 23, 2018

*Corresponding author: Sumit Sharma, Department of E.N.T, Mayo Institute of Medical Sciences, Barabanki. (U.P.), India,

Email: entsumit@rediffmail.com

\section{Introduction}

Allergic fungal rhino sinusitis (AFRS) is an allergic response in Sino nasal tract mucosa to aerosolized fungal allergens, amplified and perpetuated by eosinophils. It is a noninvasive form of fungal rhino sinusitis with an incidence of between 6 and $9 \%$ of all rhino sinusitis requiring surgery. Regional variation in incidence has been reported [1]. It is a benign noninvasive sinus disease, believed to be an allergic reaction to aerosolized environmental fungi. It has been almost three decades when in 1976 Safirstein noted that the combination of polyposis, crust formation and sinus cultures yielding aspergillus was similar to the constellation of findings observed in allergic bronchopulmonary aspergillosis. Initially it was called as Allergic Aspergillus sinusitis but later on it was found that fungus other than Aspergillus are also involved henceit was changed to Allergic fungal sinusitis. It is probably the most frequently occurring fungal rhino sinusitis disorder [2]. AFRS was first recognized in the late 1970 s by pulmonologists and pathologists who noted the distinct clinical and immunologic similarities that it shared with allergic broncho-pulmonary aspergillosis (ABPA) [3].

Fungal rhino sinusitis (FRS) is defined as the rhino sinusitis where fungi are responsible for causing the immunepathogenesis. Fungal infections involving para-nasal sinuses can be classified under two broad categories. 1. Non invasive - Saprophytic infections, fungal ball, allergic fungal sinusitis 2 . Invasive - Acute fulminant invasive sinusitis, chronic invasive sinusitis and granulomatous invasive fungal sinusitis [4]. Clinically 6 different types of fungal sinusitis have been described depending on the pathophysiology and clinical features:
a) Acute fulminant invasive fungal sinusitis
b) Chronic invasive fungal sinusitis
c) Granulomatous invasive fungal sinusitis
d) Fungal ball
e) Allergic fungal rhino sinusitis
f) Eosinophilic fungal rhino sinusitis [4].

In the present discussion we will discuss about the Clinical manifestations, Diagnostic criteria's, histopathological criteria's, radiological criteria's of the disease along with management.

\section{Clinical Presentation}

The incidence of AFRS appears to vary by geographical region. Review of the world's literature reveals that the majority of sites reporting cases of AFRS are located in more temperate regions where relative humidity is high [3]. The clinical presentation is nonspecific and may be similar to those of other forms of chronic sinusitis. They present with progressive nasal obstruction, crusting, rhinorrhoea, and chronic rhino sinusitis with nasal polyposis and viscid, dark mucoid discharge with greenish black nasal casts not responding to medical or surgical therapy [5]. Pain is uncommon in patients with AFS and when present suggests the concomitant presence of bacterial rhino sinusitis [6]. A patient with AFRS is usually an immune-competent atopic young adult or an adolescent, and less commonly a child, though the disease has been found in all ages [5]. Children usually present with unilateral disease $(70 \%$ cases) while only $37 \%$ adults have one-sided presentation Patro et al. [7] observed AFRS in children to be more aggressive with higher fungal load and less response to treatment as compared to adults. In general, $66 \%$ of AFRS patients have a history of allergic rhinitis, $90 \%$ demonstrate increased specific IgE to one or more fungi, and around $50 \%$ suffer from asthma [8]. No linkage to aspirin sensitivity has been established [3]. The presence of Nasal polyposis has been reported to be up to $100 \%$ [1] Most of these patients also show multiple previous sinus surgeries.

Complications of AFRS include [5]

a) Occular: Visual disturbances, proptosis, telecanthus,

b) Facial deformity

c) Neuropathies

d) Intracranial abscess.

e) Bony erosion is observed in the majority of cases belonging to a young age group. It probably occurs due 
to blockage of ostia of the sinuses by polyposis leading to expansion of sinuses. Commonly, the ethmoid sinus is affected with lesion extending to orbit (especially lamina papyracea) and the anterior cranial fossa. Expansion, remodeling, or thinning of involved sinus walls was common and was thought to be due to the expansile nature of the accumulating mucin.

In contrast to the often subtle symptoms of AFRS, physical findings are often more remarkable. The range of physical findings on examination is typically broad, ranging from nasal airway obstruction resulting from intranasal inflammation and polyposis to gross facial disfigurement and orbital or ocular abnormalities (3) and complications mentioned above.

\section{Diagnostic Criterias}

The most widely accepted criteria for the diagnosis is the Bent \& Kuhn diagnostic criteria for RFRS Table 1.

Table 1: Diagnostic criteria for afrs by bradley f. Marple [3].

\begin{tabular}{|c|c|c|}
\hline & Major Criteria's & Minor criteria's \\
\hline 1 & Type I hypersensitivity confirmed by history, skin tests, or \\
serology & Asthma \\
\hline 2 & Characteristic CT scan (double density sign) & Unilateral predominance \\
\hline 3 & Eosinophilic mucus without fungal invasion into sinus tissue. & Fungal culture \\
\hline 4 & Positive fungal stain of sinus contents removed intraoperative or \\
during office endoscopy & Charcot Leyden crystals \\
\hline 6 & Immuno-competence (replaces no 1 at St Paul's Sinus Centre) & Serum Eusinophilia. \\
\hline
\end{tabular}

\section{Swain's modification of Bent's criteria [4]}

de Shazo and Swain slightly modified the diagnostic criteria laid out by Bent. They left out the type I hypersenitivity criteria

\begin{tabular}{|c|c|c|c|}
\hline A. & Symptoms Requires one of the following: & B. & Objective findings Requires all of the following: \\
\hline 1. & Anterior and/or posterior nasal drainage & 1. & Presence of allergic mucin (pathology showing fungal hyphae \\
\hline 2. & Nasal obstruction & & with degranulating eosinophils) \\
\hline 3. & Decreased sense of smell & 2. & Evidence of fungal specific IgE (skin test of in vitro test) \\
\hline 4. & Facial pain-pressure-fullness & 3. & No histologic evidence of invasive fungal disease \\
\hline C. & Radiographic Findings Highly recommended: & B. & Objective findings Requires all of the following: \\
\hline 1. & Sinus CT demonstrating & 1. & Presence of allergic mucin (pathology showing fungal hyphae \\
\hline 2. & Bone erosion & & with degranulating eosinophils) \\
\hline 3. & Sinus expansion & 2. & Evidence of fungal specific IgE (skin test of in vitro test) \\
\hline 4. & Extension of disease into adjacent anatomic areas & 3. & No histologic evidence of invasive fungal disease \\
\hline
\end{tabular}

\section{Pitfalls to be avoided in the diagnosis of AFRs are}

a) The failure to recognize that the presence of classic allergic mucin, but without fungal involvement, can also be seen patients with the non-AFS disorder 'eosinophilic mucin rhino sinusitis' (EMRS).

b) Diagnostic confusion can be minimized by avoiding the temptation to culture trans nasal secretions in the office, the results of which include the presence of normal nasal flora.

\section{Histopathological Criterias}

The diagnosis of AFRS is histopathological. We will discuss this in two heads - The allergic mucin and the fungus. alone since only $2 / 3$ of patients with allergic fungal sinusitis manifested with hypersensitivity to fungal protein in various studies Table 2.

\section{The Allergic Mucin}

It is the production of allergic mucin that is unique to AFRS, and serves as a hallmark of the disease. Grossly, allergic fungal mucin is thick, tenacious, and highly viscous in consistency; its color may vary from light tan to brown or dark green. This characteristic gross appearance has led to the use of such descriptive terms as 'peanut-butter' and 'axlegrease'. Given both the location of this mucin within the para nasal sinuses as well as its thick consistency, it is common that allergic fungal mucin is initially encountered at the time of surgery. As a result of this, the diagnosis of AFRS is frequently delayed until such time [3]. Microscopically hematoxylin and eosin (H\&E) staining shows eosinophilic mucin in the form of onion laminations 
of eosinophils and their degradation products in the center surrounded by light-stained mucin and Charcot Leyden crystals. Polypoid mucosa is edematous with inflammatory mixture of lymphocytes, eosinophils, and plasma cells. Routine H\&E staining shows hyphae as a negative image as fungus fails to stain with H\&E and are detected in $67.5 \%$ of AFRS cases. The morphology of hyphae may be distorted, swollen, and have central pallor [5]. Given that fungal hyphae are frequently rare, scattered, and fragmented within allergic mucin, identification is extremely difficult unless specific histologic stains are used [3]. The specimen for the testing of allergic mucin must be collected preoperatively and not as a nasal lavage to avoid a false positive test as well as to rule out isolating normal non pathological fungal flora of the nose.

\section{The Fungus}

Since initially aspergillous was isolated from many samples of allergic mucin hence it was initially labeled as "Allergic Aspergillus Sinusitis." However, the term AAS was changed to "allergic fungal sinusitis" when etiologic agents other than Aspergillus spp. (dematiaceous group including Bipolaris spp., Alternaria spp., Curvularia spp.) were identified [5]. Fungal elements are recognized for a unique ability to absorb silver. This property is the basis for various silver stains, such as Fontana Masson stains and Grocott's or Gomori's methamine silver (GMS) stain, which turn fungi black or dark brown. The use of a fungal stain complements the findings of initial H\&E stain, and is extremely important in the identification of fungi. Fungal cultures of allergic fungal mucin may provide some supportive evidence helpful in the diagnosis and subsequent treatment of AFRS, but must be interpreted with caution. It is important to realize that the diagnosis of AFRS is not established, nor eliminated based upon the results of these cultures. The variable yield of fungal cultures (64-100\%) renders AFRS in the presence of a negative fungal culture quite possible. Conversely, a positive fungal culture fails to confirm the diagnosis of AFRS, as it may merely represent the presence of saprophytic fungal growth. It is for this reason that the histologic appearance of allergic mucin remains the most reliable indicator of AFRS [3].

Note: Bacterial cultures or staining of this allergic mucin may detect the presence of Staphylococcal aureus [5].

\section{Radiological Criterias}

The radiological features of Allergic Fungal sinusitis are quite characteristic and radiology must always be done in all suspected cases. Bothe CT scan and MRI have distinctive features like:

\section{CT Scan}

Dr. Bruno Di Muzio (1) suggested that the majority of sinuses show near complete opacification. On unenhanced CT, the sinuses are typically opacified by centrally (often serpiginous) hyper dense material with a peripheral rim of hypo dense mucosa.
Approximately $40 \%$ of patients may have each of the following features 4 :
a) Expansion of an involved sinus
b) Remodeling and thinning of the bone sinus walls
c) Erosion of the sinus wall

Am Fam [9] suggested that the degree of bony erosion and extension beyond a sinus may mimic aggressive sinonasal neoplasms. The presence of increased internal attenuation on unenhanced CT scans may help to distinguish allergic fungal sinusitis from an invasive tumor. The presence of expansion and thinning of the sinus walls may help separate allergic fungal sinusitis from chronic sinusitis of other origins. He also suggested that mucosal thickening was visualized in any of the sinuses, but the ethmoid sinus complex was the most commonly involved sinus. At least one ethmoid sinus complex demonstrated infection in $96 \%$ of the patients. And the next most commonly involved region was the maxillary sinus, which harbored infection in $93 \%$ of patients Table 3.

Table 3.

\begin{tabular}{|c|c|}
\hline 1 & $\begin{array}{c}\text { Complete opacification of at least one paranasal } \\
\text { sinus }\end{array}$ \\
\hline 2 & Expansion of involved sinuses \\
\hline 3 & $\begin{array}{c}\text { Attenuation/erosion of the bone bordering involved } \\
\text { sinus }\end{array}$ \\
\hline 4 & Heterogeneity of signal within involved sinus \\
\hline 5 & Unilateral or asymmetric distribution of disease \\
\hline 6 & Displacement of adjacent anatomic compartments \\
\hline
\end{tabular}

Note: Bradley F Marple [3] suggested the following CT findings associated with AFRS.

Cade Martin [10] suggested that in non-contrast CT high attenuation allergic mucin within lumen of sinuses can mimic a mucocele with expansion of the sinus. Arunaloke Chakrabarti [5] described CT findings as consisting of multiple sinus opacifications with central hyper-attenuation (central serpiginous or starry sky appearance), sinus mucocele, skull base erosions (56\% of AFRS patients versus 5\% of non AFRS patients) and remodeling with a "pushing border" at skull base.

He also suggested that Bone erosion and extension of disease into adjacent anatomic areas was encountered in $20 \%$ of the patients and was more likely to occur in the presence of bilateral, advanced disease. Expansion, Remodeling, or thinning of involved sinus walls was thought to be due to the expansile nature of the accumulating mucin. Desiccation of sinus contents may also contribute to the hyperdense areas seen on CT scans. Although these findings are not specific for AFRS, they are relatively characteristic of the disease, and may provide preoperative information suggestive of a diagnosis of AFRS. 
MRI

Dr. Bruno Di Muzio [11] suggested that Hypo intensity on T1WI and T2WI is the most common finding. T1: hypo intense inflamed mucosal thickness. It can have multiple T1 appearances. T2: usually a hyper intense peripheral inflamed mucosal thickness, He suggested that low T2 signal or signal void is due to high concentration of various metals such as iron, magnesium and manganese concentrated by fungal or Nganisms as well as high protein and low free water content in allergic mucin. T1 C+ (Gd): an inflamed mucosal lining has contrast enhancement -- no enhancement in the center or majority of the sinus contents (c.f. neoplasms) Table 4.

Table 4.

\begin{tabular}{|c|c|}
\hline Stage 0 & No evidence of disease. \\
\hline Stage 1 & $\begin{array}{c}\text { Odematous mucosa / allergic } \\
\text { mucin }\end{array}$ \\
\hline Stage 2 & $\begin{array}{c}\text { Polypoidal mucosa / allergic } \\
\text { mucin }\end{array}$ \\
\hline Stage 3 & Polyps and Fungal debris \\
\hline
\end{tabular}

Manohar Aribandi et al. [12] suggested that they have frequently observed high signal intensity or mixed low, intermediate, and high signal intensity on T1-weighted images in these patients. He also stated that although the condition is not considered invasive, if left untreated, the involved sinuses expand and there is smooth bone erosion with subsequent intracranial or intra orbital extension and resulting cranial or orbital symptoms. Intracranial extension is usually limited by the dura to the extradural space.

Cade Martin [10] suggest that moderately high T1 signal, low T2 signal with expanded sinus can be seen in allergic fungal sinusitis, mucocele, or sino-nasal polyposis.

\section{Treatment}

The treatment of AFRS continues to evolve, most otolaryngologists now understand what constitutes AFS, but this improved recognition has not translated into treatment advances. Main treatment modalities are Surgery, oral steroids, Immunotherapy, Oral antifungals and leukotriene modulators. Most of the times a combination therapy is recommended.

\section{Surgery}

The first step in treatment for any AFS patient is paranasal sinus surgery to both remove all obstructing inspissated allergic mucin and resect all diseased hypertrophic sinus mucosa and polyps [1]. Earlier radical surgery was performed to remove the whole mucosa. Currently, endoscopic tissuesparing (conservative) technique called functional endoscopic sinus surgery (FESS) has surpassed it as the surgery of choice. The surgery also helps remove the antigenic stimulus from the sinuses. It is recommended to enlarge the maxillary sinus to the maximum possible width through the middle meatus in AFRS patients. AFRS is considered to have poor surgical outcome among all types of CRS. The FESS improves quality of life although revision surgery is required in $15 \%-20 \%$ patients [5]. The factors contributing to need of revision surgery are poor drainage of the frontal recess or the frontal sinus neo-ostium due to the presence of remains of the uncinate process and anterior ethmoid cells, a missed maxillary sinus ostium, a lateralized middle turbinate, scarring, osteoneogenesis, or recurrent polyposis. The disease-specific measures and quality of life are predicted to be poor when the amount of mucosal eosinophilia is $>10$ eosinophils /high-power field during FESS procedure [5]. The accumulation of mucin appears to play a role in the cyclic recurrence of the disease by way of perpetuating antigenic exposure. In theory, complete surgical removal of mucin breaks this cycle [3]. Endoscopic surgery for allergic fungal sinusitis may be associated with more complications when compared to endoscopic sinus surgery for other pathologies. Extensive disease may cause spatial disorientation. There may be areas of bony dehiscence, which may confuse or distort anatomic boundaries, causing increased risk of orbital and intracranial complications. It includes penetration of dura or periorbita resulting in diplopia, blindness, intracranial hemorrhage or cerebrospinal fluid rhinorrhoea. Occasional cases of fungal invasion into adjacent tissues have also been described [2]. In addition to surgery, postoperative endoscopic cleanings should be periodically scheduled after surgery, and patients should be followed for a very long period which helps in preventing recurrence [6].

The rate of relapse in pediatric cases (ranges from 25 to $55 \%$ ) is higher than in adults. It has been hypothesized that this high rate of recurrence in the pediatric population is related to the risks associated with long-term or repetitive systemic steroid therapy. However, patients with recurrence should undergo surgical revision [6]. Patients should be followed up for a long time and post treatment mucoal status described by Kupferberg etal (endoscopic staging system of Allergic Fungal sinusitis) must be used to evaluate patients as given below.

\section{Oral Corticosteroids}

Similar to surgical therapy, oral steroids is the mainstay of management of AFRS and have a significant role postoperatively in reducing recurrence and inflammatory markers, and ultimately improving the outcome in these patients. They may even obviate the need of revision surgery [5]. Most experts now agree that adding OCS to sinus surgery gives the best outcomes. But there is no uniformity in optimal dosing regimen and length of therapy. Kuhn and Javer recommend oral prednisolone starting with $0.4 \mathrm{mg} / \mathrm{kg}$ body weight postoperatively and slowly tapering it to $0.2 \mathrm{mg} / \mathrm{kg}$ body weight. After maintaining normal mucosa for four months period, the dose is reduced to $0.1 \mathrm{mg}$ / $\mathrm{kg}$ body weight for another two months and stopped. Landsberg et al. [2]. Demonstrated the radiologic and endoscopic benefits of preoperative administration of oral steroids in AFRS patients. Their use in preoperative period helps in removing mechanical 
obstruction and that helps in viewing sin nasal anatomy during FESS [13] although the steroids have shown significant benefit in AFRS patients, their prolonged use is associated with adverse effects. On the contrary, topical corticosteroids possess a better safety profile and have shown benefit in the form of decreased polyp size and recurrence when added to local saline irrigation. (5) However we must understand that essentially, steroids act by blunting the pathologic hypersensitivity to fungal antigens, but they do not permanently reverse the disease process, leaving a great need for other forms of therapy.

\section{Oral Anti Fungals}

There is a lack of evidence for any recommendation of oral or topical antifungal agents for AFRS. Since the disease is noninvasive and the fungus lies in the allergic mucin, systemic antifungals should be ineffective against the fungi, which are located extramucosally, outside the range of the drug circulation. Thus in order to produce an effect, a systemic antifungal must be secreted in sinus mucus, a phenomenon that has not been supported and probably does not occur. (14) A study by Kuhn and colleagues showed amphotericin B and ketoconazole to be most effective agents in-vitro [2]. However it may be considered as an option in post-surgical refractory patients and they may provide benefit in terms of reduction of symptoms, steroid dependence, and tendency of recurrences such as ABPA. Kupferberg et al. noted improved endoscopic scoring when oral antifungals were administered to AFRS patients while decreased recurrence (around 50\%) and revision surgery (around 20\%) were reported by Rains and Mineck using oral itraconazole [3] Systemic antifungals such as amphotericin B play no role in AFS Jen et al. [14] also supported the benefits of a topical antifungal medication. However, the benefits of antifungal use still need to be assessed over the adverse effects associated with systemic therapy.

\section{Immunotherapy}

With evidence supporting fungal hypersensitivity in the pathogenesis of AFRS, fungal immunotherapy appeared a strong potential for benefit. Initial studies revealed not only that this therapy is safe, but surprisingly led to a remarkable decrease in disease recurrence. In one such study, patients treated with fungal immunotherapy for a period of three years following surgery showed a significant decrease in their rate of recurrence and also had less mucosal edema as noted on endoscopy and reported better quality of life [3].

Previously it was contraindicated because it was thought that antigens administered could provoke a Gel and Coomb type III reaction worsening the patient's condition. Recently it was shown that since surgery is able to remove the inciting fungal load from the Para nasal sinuses. Therefore immunotherapy might achieve sufficient immunomodulation to benefit the patient. A study conducted by Mabry et al showed immunotherapy can reduce the reliance on the systemic and topical steroids as well.
Although Marple et al. [2] in 2002 failed to show any significant benefit of immunotherapy, thereby questioning its role in management. Since subcutaneous form of Immunotherapy is not associated with any side effect Therefore, immunotherapy may serve as adjunct therapy in patient's refractory to surgery and antifungal therapy.

\section{Leukotriene Modulators}

There is no controlled study available regarding use of these agents in AFRS. There is only one case report of successful postoperative management of AFRS with montelukast $10 \mathrm{mg}$ daily along with topical corticosteroids. However, these agents have shown mixed results and no benefit in comparison to steroids [5].

\section{Conclusion}

Allergic fungal rhino sinusitis (AFRS) is an allergic response of the Sino nasal tract mucosa to aerosolized fungal allergens, and is a noninvasive form of fungal rhino sinusitis. It is now recognized to be a distinct subgroup of the common hypertrophic rhino sinusitis disorders. It is a unique entity with a great deal of controversy in classification, pathogenesis, diagnostic criteria, and management protocols. The diagnosis of AFRS although straightforward which combines clinical, radiological, microbiological, and pathological criterias, presentation sometimes can be confusing. Multimodality treatment with surgery and Oral steroids is the mainstay of the treatment with antifungals and Immune-modulators being used in special circumstances. Since the diagnosis and treatment modalities are being understood, more studies are needed to enable improved postoperative results.

\section{References}

1. Mark S Schubert (2009) Allergic fungal sinusitis: pathophysiology, diagnosis and management. Med Mycol 47(Supplement 1): S324-S330.

2. Navneet Kumar, Vandana Berry (2008) Allergic Fungal Sinusitis. JK Science $10(1)$.

3. Bradley F Marple (2006) Allergic fungal rhino sinusitis: A review of clinical manifestations and current treatment strategies. Medical Mycology 44: S277-S284.

4. Balasubramanian Thiagarajan Geetha Ramamoorthy (2013) Fungal sinusitis An Overview. otolaryngology 3(2).

5. Arunaloke Chakrabarti, Harsimran Kaur (2016) Allergic Aspergillus Rhinosinusitis. J Fungi 2: 32.

6. Berlucchi, Pedruzzi (2012) Allergic Fungal Sinusitis in Children. J Aller Ther S5.

7. Thorp BD, McKinney KA, Rose AS, Ebert CS (2012) Allergic fungal sinusitis in children. Otolaryngol Clin North Am 45(3): 631-642.

8. Patadia MO, Welch KC (2015) Role of immunotherapy in allergic fungal rhino sinusitis. Curr. Opin. Otolaryngol. Head Neck Surg 23(1): 21-28.

9. (1998) Features of Allergic Fungal Sinusitis on CT Scan. Am Fam Physician 58(4): 980.

10. Cade Martin Fungal Sinusitis: An Overview. 
11. Bruno Di Muzio, Wael Nemattalla Allergic fungal sinusitis.

12. Imaging Features of Invasive and Noninvasive Fungal Sinusitis: A Review

13. Landsberg R, Segev Y, De Rowe A, Landau T, Khafif A, Fliss DM (2007) Systemic corticosteroids for allergic fungal rhinosinusitis and chronic

This work is licensed under Creative Commons Attribution 4.0 License

DOI: $10.19080 /$ GJ0.2018.13.555865 rhinosinusitis with nasal polyposis: A comparative study. Otolaryngol Head Neck Surg 136(2): 252-257.

14. John P Bent, Frederick A Kuhn MD (1996) Allergic Fungal Sinusitis/ Polyposis. Allergy and Asthma Proc 17(5): 259-298.

\section{Your next submission with Juniper Publishers} will reach you the below assets

- Quality Editorial service

- Swift Peer Review

- Reprints availability

- E-prints Service

- Manuscript Podcast for convenient understanding

- Global attainment for your research

- Manuscript accessibility in different formats ( Pdf, E-pub, Full Text, Audio)

- Unceasing customer service

Track the below URL for one-step submission https://juniperpublishers.com/online-submission.php 